\title{
Aspergillus flavus
}

National Cancer Institute

\section{Source}

National Cancer Institute. Aspergillus flavus. NCI Thesaurus. Code C77181.

A species of mold fungus in the phylum Ascomycota that is associated with asperg illosis of the lungs. 